\title{
DECOMPOSITION THEORY FOR LATTICES WITHOUT CHAIN CONDITIONS
}

\author{
BY \\ R. P. DILWORTH AND PETER CRAWLEY
}

1. Introduction. The classical structure theorems of algebraic systems usually assume some type of finiteness condition. The most common finiteness restriction is a chain condition. Thus the proofs of the fundamental structure and decomposition theorems for lattices have customarily required the ascending chain condition. Moreover, these theorems generally fail to hold in arbitrary lattices. Nevertheless, there are important examples of decomposition theorems for lattices associated with abelian groups and rings in which the ascending chain condition does not hold. These lattices have in common another distinctive property-they are compactly generated. Namely, the lattice is generated by a collection of elements which are finitely dependent in the sense that any such element is contained in the union of a set of lattice elements if and only if it is contained in the union of a finite subset. The compact elements of the lattice of ideals of a ring are the finitely generated ideals. Likewise the compact elements of a lattice of congruence relations are the congruence relations generated by collapsing a finite collection of element pairs. More generally, the lattice of congruence relations and the lattice of subsystems of a universal algebra are compactly generated. Since structure theorems for an algebraic system correspond to decomposition theorems in the lattice of congruence relations, this strongly suggests that compactly generated lattices are the appropriate domain in which to study decomposition theory. Furthermore, since every lattice satisfying the ascending chain condition is trivially compactly generated, it follows that the classical case will be subsumed in the more general theory.

In the present paper we shall make the further restriction that the lattice is atomic, that is, that every quotient contains minimal elements. Thus this case generalizes the finite dimensional theory (Dilworth $[2 ; 3]$ ) in that the ascending chain condition is replaced by compact generation and the descending chain condition, by atomicity. Now the basic technique in the classical case consisted in establishing a relationship between the properties of the decompositions of an element $a$ of the lattice and the structure of the finite dimensional quotient lattice generated by the elements covering $a$. In the present case, the quotient lattice is no longer finite dimensional and the decompositions are no longer finite. Nevertheless, compact generation and atomicity imply sufficient regularity in the structure of the quotient lattices

Received by the editors September 1, 1959. 
and the decompositions that a relationship can be established which preserves many of the properties of the finite dimensional case.

It should be noted that there are many important examples of compactly generated atomic lattices which do not satisfy the ascending chain condition. Some of these examples are the lattice of subgroups of an infinite torsion abelian group, the lattice of congruences of a weakly atomic modular lattice, the lattices of subspaces of an infinite dimensional vector space, and any infinite dimensional exchange lattice.

The decomposition theory for nonatomic compactly generated lattices requires a quite different approach and will be treated by one of us elsewhere.

2. Preliminaries. Lattice elements will be denoted by lower case latin letters while sets of lattice elements will be denoted by latin capitals. If $a$ is an element of the lattice $L$ and $S$ is a subset of $L, a \cap S$ will denote the set of elements $a \cap s$ where $s \in S . a \cup S$ is similarly defined. The covering relation in $L$ will be denoted by $a>b . S \wedge T$ and $S \vee T$ will denote set-intersection and union respectively.

DEFinition 2.1. An element $c$ of a lattice $L$ is said to be compact if $c \leqq U S$ implies $c \leqq U S^{\prime}$ for a finite subset $S^{\prime}$ of $S$.

If $c_{1}$ and $c_{2}$ are compact, then $c_{1} \cup c_{2} \leqq U S$ implies $c_{1} \leqq U S$ and $c_{2} \leqq U S$. Hence there exist finite subsets $S_{1}$ and $S_{2}$ of $S$ such that $c_{1} \leqq U S_{1}$ and $c_{2} \leqq U S_{2}$. But then $c_{1} \cup c_{2} \leqq \bigcup\left(S_{1} \bigvee S_{2}\right)$. Thus we have the following lemma.

Lемма 2.1. The compact elements of a lattice are closed under finite union.

The compact elements of $L$ will be denoted by $C(L)$.

Definition 2.2. A lattice $L$ is compactly generated if $L$ is complete and $a=\bigcup\{c \in C(L) \mid c \leqq a\}$ for all $a \in L$.

A complete lattice is thus compactly generated if the compact elements generate the lattice under unrestricted joins. If every element is compact, the lattice is trivially compactly generated.

Lemma 2.2. Let $A$ be an ideal of a complete lattice $L$. Then if $\cup A$ is compact, $A$ is principal.

For if $c=\cup A$ is compact, then $c \leqq \cup S^{\prime}$ where $S^{\prime}$ is a finite subset of $A$. But then $c \in A$ and hence $A=(c)$.

COROLLARY. Every element of a complete lattice $L$ is compact if and only if $L$ satisfies the ascending chain condition.

The following lemmas develop some of the properties of lattices satisfying the ascending chain condition which also hold in compactly generated lattices.

LEMMA 2.3. Every compactly generated lattice is join continuous; that is, $a \cap \cup B=U(a \cap B)$ for every ideal $B$ of $L$.

For let $c \leqq a \cap \cup B$ where $c \in C(L)$. Then $c \leqq \cup B$ and hence $c \leqq \cup B^{\prime}$ where 
$B^{\prime}$ is a finite subset of $B$. But then $c \in B$ and hence $c=a \cap c \leqq U(a \cap B)$. Thus $a \cap \cup B=\mathrm{U}\{c \in C(L) \mid c \leqq a \cap \cup B\} \leqq \mathrm{U}(a \cap B)$. Since $a \cap \cup B \geqq \cup(a \cap B)$ holds trivially, it follows that $a \cap \cup B=\mathrm{U}(a \cap B)$.

Definition 2.3. An element $q$ of a lattice $L$ is completely meet irreducible if for all $S \subseteq L, q=\cap S$ implies $q \in S$.

Clearly every completely meet irreducible element is meet irreducible. For atomic $\left({ }^{1}\right)$ lattices the two concepts coincide.

Lemma 2.4. Every meet irreducible element of a complete atomic lattice is completely meet irreducible.

For if $q$ is a meet irreducible element, let $q=\cap S$ and suppose that $s>q$ all $s \in S$. Then by atomicity there exist $p$ such that $p>q$. If $s \geqq p$ for some $s \in S$, then $s \cap p=q$ contrary to the meet irreducibility of $q$. Thus $s \geqq p$ all $s \in S$ and hence $q=\cap S \geqq p$ contrary to $p>q$. It follows that $q=s \in S$ for some $s$ and hence $q$ is completely meet irreducible.

LeMma 2.5. If $L$ is a compactly generated lattice and $a, b$ are elements of $L$ such that $a \pm b$, then there exists a completely meet irreducible element $q$ such that $a \leqq q$ and $b \$ q$.

For since $a \geq b$ and $b=U\{c \in C(L) \mid c \leqq b\}$, there exists a compact element $c \leqq b$ such that $c \leqq a$. But then $c \notin(a)$ where $(a)$ is the principal ideal generated by $a$. By the Maximal Principle there exists a maximal ideal $A$ such that $a \in A$ and $c \notin A$. Let $q=\bigcup A$. If $c \leqq q$, then $c \leqq \cup A$ and hence $c \leqq \cup A^{\prime}$ where $A^{\prime}$ is a finite subset of $A$, and thus $c \in A$ contrary to assumption. It follows that $c \mid q$ and hence $b \pm q$. If $q=\cap S$ and $s>q$ all $s \in S$, then since $A \subset(s)$ we have $c \leqq s$ all $s$ by the maximal property of $A$. Hence $c \leqq \cap S=q$ contrary to $c \$ q$. Thus $q=s \in S$ for some $s$ and $q$ is completely meet irreducible.

COROLlARY. Each element of a compactly generated lattice can be represented as a meet of completely meet irreducible elements.

For if $b$ is the meet of all completely meet irreducibles containing $a$ and $b \leq a$, then according to Lemma 2.5 there would exist a completely meet irreducible $q$ such that $a \leqq q$ and $b \leqq q$ contrary to the definition of $b$.

It is evident from the corollary that a compactly generated lattice contains sufficiently many completely meet irreducibles to give substance to a decomposition theory. Indeed it is likely that the additional assumption of atomicity is sufficient to insure the existence of irredundant decompositions. However, we shall begin our study with the consideration of semimodular lattices $\left({ }^{2}\right)$.

(1) A lattice is atomic if $a>b$ implies $a \geqq c>b$ for some $c \in L$.

(2) At the present time, an adequate decomposition theory does not exist for lattices more general than semimodular lattices even in the finite dimensional case. 
3. Semimodular lattices. Throughout this section $L$ will denote a compactly generated atomic lattice. Completely meet irreducible elements of $L$ will simply be called "irreducibles." For each $a \in L$ let $P_{a}$ denote the set of elements covering $a$. We set $u_{a}=\bigcup P_{a}$, and define $u_{a} / a=\left\{x \mid a \leqq x \leqq u_{a}\right\}$.

Lemma 3.1. $u_{a} / a$ is a compactly generated atomic lattice. Furthermore the elements of $P_{a}$ are compact elements of $u_{a} / a$.

For if $x \in u_{a} / a$, then $x=\bigcup\{c \in C(L) \mid c \leqq x\}$. But then

$$
x=\bigcup\{a \cup c \mid c \in C(L), c \leqq x\} .
$$

$a \cup c$ is compact in $u_{a} / a$ since if $a \cup c \leqq \cup S$ where $S \subseteq u_{a} / a$, then $c \leqq \cup S$ and hence $c \leqq \cup S^{\prime}$ where $S^{\prime}$ is a finite subset of $S$. But then $a \cup c \leqq \cup S^{\prime}$. Since $u_{a} / a$ is a quotient lattice of an atomic lattice, it is atomic. Finally if $p>a$ and $p \geqq c$, where $a \geqq c$ and $c \in C(L)$, then $p=a \cup c$ and hence $p$ is compact in $u_{a} / a$.

Definition 3.1. An element $s \in u_{a} / a$ is a relative (meet) irreducible of $u_{a} / a$ if there exists a completely meet irreducible $q$ of $L$ such that $q \geqq a$ and $q \cap u_{a}=s$.

Corollary. Each irreducible of $u_{a} / a$ is a relative irreducible.

For if $s$ is an irreducible of $u_{a} / a$, by the corollary to Lemma 2.5, $s=\cap Q$ where $Q$ is a set of irreducible of $L$. But then $s=\cap\left(Q \cap u_{a}\right)$ and $Q_{a} \cap u_{a} \subseteq u_{a} / a$. Hence $s=q \cap u_{a}$ for some $q$ and thus $s$ is a relative irreducible.

The decompositions of an element $a$ into irreducibles in $L$ are closely related to the decompositions of $a$ into relative irreducibles in $u_{a} / a$, as indicated in the following lemma. The proof is left to the reader.

Lemma 3.2. To each decomposition $a=\cap Q$ of the element $a$ into irreducibles in $L$ corresponds the decomposition $a=\cap\left(Q \cap u_{a}\right)$ into relative irreducibles in $u_{a} / a$. The decomposition $a=\cap Q$ is irredundant if and only if the decomposition $a=\cap\left(Q \cap u_{a}\right)$ is irredundant. Furthermore each decomposition of $a$ into relative irreducibles in $u_{a} / a$ can be obtained in this manner from a decomposition into irreducibles in $L$.

By means of the correspondence of Lemma 3.2 it is frequently possible to reduce the study of decompositions in $L$ to the study of decompositions in $u_{a} / a$. This approach is particularly appropriate when the relative irreducibles of $u_{a} / a$ coincide with the irreducibles of $u_{a} / a$.

Semimodularity is defined in the usual way.

Definition 3.2. A lattice is (upper) semimodular if $a>a \cap b$ implies $a \cup b>b$.

A lattice is lower semimodular if its dual is upper semimodular. For finite dimensional lattices, upper semimodularity is equivalent to the following weaker condition.

Definition 3.3. A lattice is weakly (upper) semimodular if $a, b>a \cap b$ imply $a \cup b>a, b$. 
We will now show that these two conditions are equivalent for compactly generated atomic lattices.

Lemma 3.3. A weakly semimodular, compactly generated, atomic lattice is semimodular.

For, let $a>a \cap b$ and define a subset $W$ as follows:

$$
W=\{x \mid b \geqq x \geqq a \cap b, a \cup x>x\} .
$$

The set $W$ is nonempty since it contains $a \cap b$. Now let $X$ be a chain of $W$ and let $y=\bigcup X$. Then $b \geqq y \geqq a \cap b$. Also $a \cup y>y$, since otherwise $b \geqq y=a \cup y$ $\geqq a$ contrary to $a>a \cap b$. Let $a \cup y \geqq z>y$. Since the lattice is compactly generated there exists a compact element $c$ such that $z \geqq c, y \geqq c$. Then $c \leqq a \cup y$ $=a \cup(\cup X)$. Since $c$ is compact there exist $x_{1}, \cdots, x_{n}$ in $X$ such that $c \leqq a \cup x_{1} \cup \ldots \cup x_{n}$. Since $X$ is a chain, $x_{1} \cup \ldots \cup x_{n}=x \in X$. Hence $c \leqq a \cup x$ and thus $x \leqq c \cup x \leqq a \cup x$. If $x=c \cup x_{1}$ then $y \geqq x=c \cup x \geqq c$ contrary to $y \geq c$. Thus $x<c \cup x \leqq a \cup x$. Since $a \cup x>x$ we have $c \cup x=a \cup x \geqq a$. But then $z \geqq c \cup y \geqq c \cup x \geqq a$ and hence $z \geqq a \cup y$. Thus $z=a \cup y$ and hence $a \cup y>y$. It follows that $\mathrm{U} X=y \in W$. By the Maximal Principle $W$ contains a maximal element $x_{0}$. Suppose $b>x_{0}$. Then by atomicity, $b \geqq p>x_{0}$ for some $p$. Since $b \geq a$ it follows that $p \neq a \cup x_{0}$. Also $a \cup x_{0}>x_{0}$ since $x_{0} \in W$. Hence $p \cap\left(a \cup x_{0}\right)$ $=x_{0}$ and by weak semimodularity we have $a \cup p=\left(a \cup x_{0}\right) \cup p>p$. But $b \geqq p \geqq a \cap b$ and hence $p \in W$ contrary to the maximal property of $x_{0}$. Thus $b=x_{0}$ and hence $a \cup b=a \cup x_{0}>x_{0}=b$.

The following generalization of a well known characterization of finite dimensional modular lattices will be needed.

Lemma 3.4. A compactly generated atomic lattice which is both upper and lower semimodular is modular.

For let $a \geqq b$ and let $V$ be defined as follows:

$$
V=\{x \mid b \cup(a \cap c) \geqq x \geqq a \cap c, x=a \cap(x \cup c)\} .
$$

$V$ is nonempty since it contains $a \cap c$. Now let $X$ be a chain of $V$ and let $y=\bigcup X$. Clearly $b \cup(a \cap c) \geqq y \geqq a \cap c$. Also, $a \cap(y \cup c)=a \cap \cup(X \cup c)$ $=\bigcup(a \cap(X \cup c))=\cup X=y$ since the lattice is continuous. Hence $y \in V$ and thus every chain in $V$ has a bound in $V$. By the maximal principle $V$ contains a maximal element $x_{0}$. If $b \cup(a \cap c)>x_{0}$, there exists $p$ such that $b \cup(a \cap c)$ $\geqq p>x_{0}$. Now $x_{0} \cup c \geq p$ since otherwise $x_{0}=a \cap\left(x_{0} \cup c\right) \geqq p$. Thus $p \cap\left(x_{0} \cup c\right)$ $=x_{0}<p$. By upper semimodularity we have $p \cup c=p \cup\left(x_{0} \cup c\right)>x_{0} \cup c$. Now $x_{0} \cup c \geqq a \cap(p \cup c)$ since otherwise $x_{0}=a \cap\left(x_{0} \cup c\right) \geqq a \cap(p \cup c) \geqq p$. Hence $[a \cap(p \cup c)] \cup\left(x_{0} \cup c\right)=p \cup c>x_{0} \cup c$. By lower semimodularity we have

$$
a \cap(p \cup c)>a \cap(p \cup c) \cap\left(x_{0} \cup c\right)=a \cap\left(x_{0} \cup c\right)=x_{0} .
$$

But $a \cap(p \cup c) \geqq p>x_{0}$ and hence $a \cap(p \cup c)=p$. Thus $p \in V$ contrary to the maximal property of $x_{0}$. It follows that $x_{0}=b \cup(a \cap c)$ and hence 


$$
b \cup(a \cap c)=x_{0}=a \cap\left(x_{0} \cup c\right)=a \cap(b \cup(a \cap c) \cup c)=a \cap(b \cup c)
$$

We thus conclude that the lattice is modular.

Definition 3.4. A subset $N$ of $P_{a}$ is independent if $p \cap U(N-p)=a$ for all $p \in N$.

Lemмa 3.5. A subset $N$ of $P_{a}$ is independent if and only if every finite subset of $N$ is independent.

If $N$ is not independent, then for some $p \in N$ we have $p \geqq p \cap U(N-p)>a$ and hence $p=p \cap U(N-p) \leqq U(N-p)$. Since $p$ is compact in $u_{a} / a$ we have $p \leqq \mathrm{U}\left(N^{\prime}-p\right)$ where $N^{\prime}$ is a finite subset of $N$ containing $p$. Thus $N^{\prime}$ is not independent. Conversely, it is clear that any finite subset of an independent set is independent.

LEMMA 3.6. Let $a$ be an element of a semimodular, compactly generated, atomic lattice and let $N$ be an independent subset of $P_{a}$. Then $N$ is a maximal independent subset of $P_{a}$ if and only if $U N=u_{a}$.

If $U N=u_{a}$, then $N$ is clearly a maximal independent subset of $P_{a}$. Now let $N$ be maximal and let $p \in P_{a}$. Then either $U N \geqq p$ or there exists $p^{\prime} \in N$ such that $U\left(N-p^{\prime}\right) \cup p \geqq p^{\prime}$. In the second case $U\left(N-p^{\prime}\right) \geqq p$ since otherwise $\mathrm{U}\left(N-p^{\prime}\right) \geqq p^{\prime}$ contrary to the independence of $N$. Thus we have $\mathrm{U}\left(N-p^{\prime}\right)$ $\cup_{p}>\cup\left(N-p^{\prime}\right)$ by semimodularity. But $U\left(N-p^{\prime}\right) \cup p \geqq \cup\left(N-p^{\prime}\right) \cup p^{\prime}=U(N)$ $>\bigcup\left(N-p^{\prime}\right)$. Thus $U(N)=\bigcup\left(N-p^{\prime}\right) \cup p \geqq p$. Hence $U N \geqq p$ in either case. Since $p$ was an arbitrary element of $P_{a}$ we have $U N \geqq \cup P_{a}=u_{a}$.

LEMMA 3.7. Let $a$ be an element of a semimodular, compactly generated, atomic lattice $L$ and let $N$ be an independent subset of $P_{a}$. Then the elements of $L$ which are joins of subsets of $N$ form a complete sublattice of $L$ which is isomorphic with the lattice of all subsets of $S$.

Consider the mapping $S \rightarrow \cup S$. If $\cup S_{1}=\cup S_{2}$, then $p_{1} \leqq \cup S_{1} \leqq \cup S_{2}$ all $p_{1} \in S_{1}$. Since $N$ is independent it follows that $p_{1} \in S_{2}$ all $p_{1} \in S_{1}$. Thus $S_{1} \subseteq S_{2}$ and similarly $S_{2} \subseteq S_{1}$. The mapping is thus one-to-one. Now let $S_{\alpha}$ be a collection of subsets of $N$. Clearly $\mathrm{U}_{\alpha}\left(\mathrm{U} S_{\alpha}\right)=\mathrm{U}\left(\mathrm{V}_{\alpha} S_{\alpha}\right)$ and hence the mapping preserves joins. Let $b=\mathrm{U}\left(\Lambda_{\alpha} S_{\alpha}\right)$ and let $T_{\alpha}=\left\{b \cup p \mid p \in S_{\alpha}-\Lambda_{\alpha} S_{\alpha}\right\}$. Then $p^{\prime}>b$ for each $p^{\prime} \in T_{\alpha}$ since $N$ is independent and $L$ is semimodular. Also $\bigcup T_{\alpha}=b \cup U\left(S_{\alpha}-\Lambda_{\alpha} S_{\alpha}\right)=\bigcup\left(\Lambda_{\alpha} S_{\alpha}\right) \cup U\left(S_{\alpha}-\Lambda_{\alpha} S_{\alpha}\right)=\bigcup\left(S_{\alpha}\right)$. Now $b \cup p_{1}=b \cup p_{2}$ where $p_{1}, p_{2} \in S_{\alpha}-\Lambda_{\alpha} S_{\alpha}$ implies that $p_{1}=p_{2}$ since $N$ is independent. Thus $\bigwedge_{\alpha} T_{\alpha}=\varnothing$. Let us suppose that $\bigcap_{\alpha}\left(U T_{\alpha}\right)>b$. Then $\bigcap_{\alpha}\left(U T_{\alpha}\right) \geqq r>b$ by atomicity and hence $r \leqq \bigcup T_{\alpha}$ all $\alpha$. But $r$ is compact in $u_{b} / b$ and hence $r \leqq U T_{\alpha}^{\prime}$ for some finite subset $T_{\alpha}^{\prime}$ of $T_{\alpha}$ for each $\alpha$. Now pick a fixed $\alpha$, then by semimodularity there exists $p^{\prime} \in T_{\alpha}^{\prime}$ such that $p^{\prime} \leqq U\left(T_{\alpha}^{\prime}-p^{\prime}\right) \cup_{r}$. Since $\Lambda_{\alpha} T_{\alpha}=\varnothing$ there exists $\beta$ such that $p^{\prime} \notin T_{\beta}$. But then $r \leqq \cup T_{\beta}$ implies $p^{\prime} \leqq \cup\left(T_{\alpha}^{\prime}-p^{\prime}\right) \cup U T_{\beta}$ $\leqq U\left(T_{\alpha} \vee T_{\beta}-p^{\prime}\right)$. If $p^{\prime}=b \cup p$, then we have $p \leqq \cup\left(S_{\alpha} \vee S_{\beta}-p\right)$ contrary to 
the independence of $N$. Hence we conclude that $\bigcap_{\alpha}\left(U S_{\alpha}\right)=\bigcap_{\alpha}\left(U T_{\alpha}\right)=b$ $=\mathrm{U}\left(\Lambda_{\alpha} S_{\alpha}\right)$. Thus the mapping preserves meets and the proof of the lemma is complete.

COROllary. If $a$ is an element of a semimodular, compactly generated, atomic lattice, then $u_{a} / a$ is complemented and each element of $u_{a} / a$ is a meet of elements covered by $u_{a}$.

For if $b \in u_{a} / a$, let $S$ be a maximal independent set of points of $u_{a} / a$ contained in $b$. Extend $S$ to a maximal independent set of points $P$. Then $b \cup \cup(P-S)=\bigcup P=u_{a}$, and clearly $b \cap \cup(P-S)=a$ since $\cap S \cap \cap(P-S)=a$. Now $p \cup b>b$ for each $p \in P-S$. Let $P_{1} \subseteq P-S$ be such that the set $\left\{p \cup b \mid p \in P_{1}\right\}$ is maximal independent. Then if $p_{1} \in P_{1}$ we have $u_{\alpha}>\cup\left\{p \cup b \mid p \in P_{1}-p_{1}\right\}$ and $\bigcap_{p} \cup\left\{p \cup b \mid p \in P_{1}-p\right\}=b$ by Lemma 3.7.

In order to prove a sufficiently general existence theorem on irredundant decompositions it is necessary to have a criterion for subsets of such decompositions. Let then $a=\cap Q$ be an irredundant decomposition of $a$ into irreducibles and let $R \subseteq Q$. If $q \in R$, we have $\cap(R-q) \geqq \cap(Q-q)>a$. Hence there exists $p \in P_{a}$ such that $\cap(R-q) \geqq \cap(Q-q) \geqq p$. Clearly $q \geqq p$ since $q \cap \cap(Q-q)$ $=\bigcap Q=a$. We thus make the following definition:

Definition 3.5. A subset $R$ of $L$ is irredundant over $a$ if for each $r \in R$, there exists $p>a$ such that $\cap(R-r) \geqq p$ and $r \geq p$.

We can now state and prove the fundamental existence theorem on irredundant decompositions.

THEOREM 3.1. Let $a$ be an element of a semimodular, compactly generated, atomic lattice and let $R$ be a set of completely meet irreducible elements containing $a$. Then $R$ can be extended to an irredundant decomposition of $a$ if and only if $R$ is irredundant over $a$.

Proof. We have proved the necessity in the paragraph preceding Definition 3.5. To prove the sufficiency let $R$ be irredundant over $a$. Then for each $q \in R$, there exists an element $p_{q} \in P_{a}$ such that $q \geq p_{q}$ and $\cap(R-q) \geqq p_{q}$. Let $K_{1}=\left\{p_{q} \mid q \in R\right\}$ and let $K_{2}$ be a maximal independent subset of $\left\{p \in P_{a} \mid p \leqq \cap R\right\}$. Let $K=K_{1} \vee K_{2}$. Now suppose that $U\left(K-p_{q}\right) \geqq p_{q}$ for some $p_{q} \in K_{1}$. Then since $q \geqq \cap R \geqq \cup K_{2}$ and $q \geqq \cap\left(R-q^{\prime}\right) \geqq p_{q^{\prime}}$ all $q^{\prime} \in R-q$ we have $q \geqq \cup\left(K_{1}-p_{q}\right) \cup \cup K_{2} \geqq \cup\left(K-p_{q}\right) \geqq p_{q}$ contrary to $q \geqq p_{q}$. Thus $\cup\left(K-p_{q}\right) \geqq p_{q}$ all $p_{q} \in K_{1}$. Next suppose that $U(K-p) \geqq p$ for some $p \in K_{2}$. Since $p$ is compact in $u_{a} / a$ there exist finite sets $K_{1}^{\prime} \subseteq K_{1}$ and $K_{2}^{\prime} \subseteq K_{2}-p$ such that $U\left(K_{1}^{\prime} \vee K_{2}^{\prime}\right) \geqq p$. Since $K_{2}$ is independent, $K_{2}^{\prime}$ is nonempty. Replacing $K_{2}^{\prime}$ by a smaller set if necessary we may assume that for some $p_{q} \in K_{1}^{\prime}$, $U\left(K_{2}^{\prime} \vee K_{1}^{\prime}-p_{q}\right) \geq p$. By semimodularity we have $p_{q} \leqq U\left(K_{2}^{\prime} \vee K_{1}^{\prime}-p_{q}\right) \cup p$ $\leqq U\left(K-p_{q}\right)$ contrary to $p_{q} \leqq \cup\left(K-p_{q}\right)$. Thus $U(K-p) \geqq p$ all $p \in K_{2}$ and $K$ is thus an independent subset of $P_{a}$.

Now for each $p \in K_{2}$, let $q_{p}$ be an irreducible such that $q_{p} \geqq U(K-p)$, 
$q_{p} \geq p$. Such irreducibles exist by Lemma 2.5. Let $Q^{\prime}=\left\{q_{p} \mid p \in K_{2}\right\}$. Since $q_{p^{\prime}} \geqq \cup\left\{K-p^{\prime}\right\} \geqq p$ all $p^{\prime} \neq p$ in $K_{2}$ we have $\cap\left(Q^{\prime}-q_{p}\right) \geqq p$. Now let $Q=Q^{\prime} \bigvee R$. If $q \in R$, then $\cap(Q-q)=\cap(R-q) \cap \cap Q^{\prime} \geqq \cap(R-q) \cap \cup K_{1} \geqq p_{q}$. If $p \in K_{2}$, then $\cap\left(Q-q_{p}\right)=\cap R \cap \cap\left(Q^{\prime}-q_{p}\right) \geqq \cup K_{2} \cap \cap\left(Q^{\prime}-q_{p}\right) \geqq p$. Thus if we show that $\cap Q=a$ then this decomposition is a fortiori irredundant. Suppose that $\cap Q>a$. Then $\cap Q \geqq p^{\prime}$ for some $p^{\prime} \in P_{a}$. Since $\cap R \geqq \cap Q$ we have $p^{\prime} \leqq \bigcap R$ and hence $p^{\prime} \leqq \cap K_{2}$ by Lemma 3.6. Furthermore $p^{\prime} \leqq \bigcap Q^{\prime} \leqq q_{p}$ for all $p \in K_{2}$. If $p^{\prime} \leqq U\left(K_{2}-p\right)$ for some $p \in K_{2}$, then $q_{p} \geqq \cup\left(K_{2}-p\right) \cup p^{\prime}=\bigcup K_{2} \geqq p$ contrary to $q_{p} \geqq p$. Thus $p^{\prime} \leqq U\left(K_{2}-p\right)$ all $p \in K_{2}$. But then $p^{\prime} \leqq \bigcap_{p} U\left(K_{2}-p\right)=\mathrm{U}\left(\Lambda_{p}\left(K_{2}-p\right)=\mathrm{U}(\varnothing)\right.$ $=a$ by Lemma 3.7 contrary to $p^{\prime}>a$. Thus $\cap Q=a$ and the proof of the theorem is complete.

Since the null set is trivially irredundant we have

COROLLARY 1. Every element of a semimodular, compactly generated, atomic lattice has an irredundant decomposition into completely meet irreducibles.

Let $q$ be an irreducible containing $a$ such that $q \geq u_{a}$. If $R$ consists of the single element $q$, then $R$ is irredundant since $\cap(R-q)=u \geqq q$. Hence we have

COROLlaRY 2. If $a$ is an element of a semimodular, compactly generated, atomic lattice and $q$ is a completely meet irreducible element such that $q \geq u_{a}$, then there exists an irredundant decomposition $a=\cap Q$ such that $q \in Q$.

We conclude this section with the following theorem on the cardinality of maximal independent sets of $P_{a}$.

THEOREM 3.2. Let $L$ be a semimodular, compactly generated, atomic lattice. Then any two maximal independent subsets of $P_{a}$ have the same cardinality.

Proof. Let $M$ and $N$ be two maximal independent subsets of $P_{a}$. We may suppose that $|M| \leqq|N|$, where $|X|$ denotes the cardinality of a set $X$. If $N$ is finite, then $u_{a} / a$ is finite dimensional and the number of elements in any maximal independent set is simply the dimension of $u_{a} / a$. Hence $|M|$ $=|N|$. If $N$ is infinite, we have $\cup M=\cup N=u_{a}$ and hence for each $p \in M$, $p \leqq \bigcup N$. But then there exists a finite subset $N_{p} \subseteq N$ such that $p \leqq \bigcup N_{p}$. Let $S=\left\{N_{p} \mid p \in M\right\}$ and let $N^{\prime}=\bigvee_{p \in M} N_{p}$. Then $N^{\prime}$ as a subset of $N$ is independent. Moreover, $p \leqq \bigcup N^{\prime}$ for each $p \in M$ implies that $U N^{\prime}=\bigcup P_{a}=u_{a}$. Hence $N^{\prime}$ is a maximal independent subset of $P_{a}$ and thus $N^{\prime}=N$. Now for each $p, N_{p}$ is a finite subset of $N$, and since $S$ is infinite, it follows that $|S|=\left|N^{\prime}\right|=|N|$. But the mapping $p \rightarrow N_{p}$ is single valued and hence $|M| \geqq|S|=|N|$. Thus $|M|=|N|$.

4. Modular lattices. Throughout this section $L$ will denote a modular, compactly generated, atomic lattice.

For modular lattices satisfying the ascending chain condition, the principal results on irreducible decompositions concern the replacement of elements in two irredundant decompositions (Dilworth [5]). Since the decom- 
positions are finite it follows from these replacement theorems, that the number of elements in an irredundant decomposition is unique. In this section we shall extend some of these replacement theorems to compactly generated modular lattices. We shall see that there are many different classes of irredundant decompositions and that replacement properties are dependent upon the classes of decompositions involved. Moreover, the unicity of the number of elements in irredundant decompositions does not generalize completely. Thus, an example of a compactly generated modular lattice is constructed in which an element has two irredundant decompositions with different cardinalities.

We begin by showing that in modular compactly generated lattices the existence of irredundant decompositions is equivalent to atomicity.

THEOREM 4.1. Every element of a modular compactly generated $L$ lattice has an irredundant decomposition into completely irreducible elements if and only if $L$ is atomic.

Proof. We need only show that if every element of $L$ has an irredundant decomposition, then $L$ is atomic.

Suppose $a=\cap Q$ is an irredundant decomposition of $a$ into completely irreducible elements. Let $q \in Q$ and $q^{*}=\bigcap\{x \in L \mid x>q\}>q$. Then $q \cup \cap(Q-q)$ $>q$ since $\cap(Q-q)>a$, and hence $q \cup \cap(Q-q) \geqq q^{*}$. By modularity we have $q \cup\left[q^{*} \cap \cap(Q-q)\right]=q^{*} \cap[q \cup \cap(Q-q)]=q^{*}>q$, and hence $q^{*} \cap \cap(Q-q)>q$ $\cap q^{*} \cap \cap(Q-q)=a$. Hence if every element of $L$ has an irredundant decomposition, then every element of $L$ is covered by some element.

Now suppose $b>a$. From the above, $p \in L$ exists such that $p>a$. Suppose $b \geq p$. Then $b \cap p=a$. Let $\left\{x_{\alpha}\right\}$ be a chain of elements of $L$ such that $b \cap x_{\alpha}=a$ for every index $\alpha$. Then by Lemma 2.4, $b \cap \bigcup_{\alpha} x_{\alpha}=\bigcup_{\alpha} b \cap x_{\alpha}=a$. Thus by the Maximal Principle it follows that a maximal element $m$ exists such that $m \cap b=a$. Let $s \in L$ be such that $s>m$. Then $b \cap s>a$. Hence $(b \cap s) \cup m=s>m$, whence $b \cap s>b \cap s \cap m=a$. Thus under any circumstances $r \in L$ exists such that $b \geqq r>a$, and hence $L$ is atomic.

Our next theorem is a direct generalization of the classical replacement theorem.

THEOREM 4.2. If $a$ is an element of a compactly generated, atomic, modular lattice and $a=\cap Q=\cap Q^{\prime}$ are two decompositions of $a$, then for each $q \in Q$ there exists $q^{\prime} \in Q^{\prime}$ such that $a=\cap(Q-q) \cap q^{\prime}$. If the decomposition $a=\cap Q$ is irredundant, then the decomposition $a=\cap(Q-q) \cap q^{\prime}$ is also irredundant.

Proof. Let $q \in Q$. For each $q^{\prime} \in Q^{\prime}$, define $r_{q^{\prime}}$ by

$$
r_{q^{\prime}}=\cap(Q-q) \cap q^{\prime} \text {. }
$$

Then $a=\bigcap_{q^{\prime} \in Q^{\prime}} r_{q^{\prime}}$, and $a \leqq r_{q^{\prime}} \leqq \bigcap(Q-q)$ for each $q^{\prime} \in Q^{\prime}$. Now since $L$ is modular, the quotient sublattices $q \cup(\cap(Q-q)) / q$ and $\cap(Q-q) / \cap(Q-q) \cap q$ 
$=\cap(Q-q) / a$ are isomorphic. $q$ is completely irreducible in $L$ and hence $q$ is completely irreducible in the quotient $q \cup(\cap(Q-q)) / q$. Thus $a$ is completely irreducible in the quotient $\cap(Q-q) / a$. But $a=\bigcap_{q^{\prime} \in Q^{\prime}} r_{q^{\prime}}$ is a representation of $a$ as a meet of elements of $\cap(Q-q) / a$, and hence for some $q^{\prime} \in Q^{\prime}, a=r_{q^{\prime}}$ $=\cap(Q-q) \cap q^{\prime}$.

Suppose the decomposition $a=\cap Q$ is irredundant. Then $\cap(Q-q)>a$, so that if $a=\cap(Q-q) \cap q^{\prime}$ is a redundant decomposition, there exists an element $q_{1} \in Q-q$ such that $a=\cap\left(Q-\left\{q, q_{1}\right\}\right) \cap q^{\prime}$. Now in this decomposition, $q^{\prime}$ can be replaced by some $q_{2} \in Q$ giving a decomposition of $a$. But then either $a=\cap(Q-q)$ or $a=\cap\left(Q-q_{1}\right)$, contrary to the irredundance of the decomposition $a=\cap Q$. Hence the decomposition $a=\bigcap(Q-q) \cap q^{\prime}$ is also irredundant.

We note that the theorem holds in any complete modular lattice.

The corollary to Lemma 3.7 can be sharpened in the case of modular lattices to give

LemMa 4.1. The quotient $u_{a} / a$ is complemented point lattice.

For since $u_{a} / a$ is complemented and modular, it is also relatively complemented. Hence if $x \in u_{a} / a$ and $y$ is the union of the points contained in $x$, then the relative complement of $y$ in $x / a$ must be $a$ and thus $x=y$.

The following lemma relating irreducibles containing $a$ to the elements covering $a$ will be needed.

Lemma 4.2. Let $a \in L$ and let $p, p^{\prime}$ be two distinct elements covering $a$. If $q$ is an irreducible such that $q \geqq a$ and $q \geq p, p^{\prime}$, then $q \cap\left(p \cup p^{\prime}\right)>a$.

For since $q \geq p, p^{\prime}$ we have $p \cap q=p^{\prime} \cap q=a$ and hence $p \cup q>q$ and $p^{\prime} \cup q>q$. Since $q$ is irreducible it follows that $p \cup q=p^{\prime} \cup q=p \cup p^{\prime} \cup q$. Thus $\left(p \cup p^{\prime}\right) \cup q>q$ and hence $p \cup p^{\prime}>q \cap\left(p \cup p^{\prime}\right)$. Since $p \cup p^{\prime}>p>a$ it follows that $q \cap\left(p \cup p^{\prime}\right)>a$.

Definition 4.1. If $a=\bigcap Q$ is a decomposition of $a$, let

$$
H_{Q}=\left\{p \in P_{a} \mid \cap(Q-q) \geqq p \text { some } q \in Q\right\} \text { and } h_{Q}=U H_{Q} .
$$

Lemma 4.3. Let $a=\cap Q$ be a decomposition of $a$ into irreducibles. Then

(1) $H_{Q}$ is an independent subset of $P_{a}$.

(2) $H_{Q}$ contains at most one element $p$ such that $\cap(Q-q) \geqq p$.

(3) If $a=\cap Q$ is irredundant, then $H_{Q}$ contains exactly one element $p$ such that $\cap(Q-q) \geqq p$.

In order to see that (2) holds, let $\cap(Q-q) \geqq p, p^{\prime}$ where $p$ and $p^{\prime}$ are distinct. Since $a=q \cap \cap(Q-q)$ it follows that $q \geq p, p^{\prime}$ and hence $q \cap\left(p \cup p^{\prime}\right)>a$ by Lemma 4.2 which contradicts $a=q \cap \cap(Q-q) \geqq q \cap\left(p \cup p^{\prime}\right)$. Now if $\cap(Q-q)$ $\geqq p$ and $p^{\prime} \in H_{Q}-p$, then $\cap(Q-q) \geqq p^{\prime}$ and hence $\cap\left(Q-q^{\prime}\right) \geqq p^{\prime}$ where $q^{\prime} \neq q$. But then $q \geqq \cap\left(Q-q^{\prime}\right) \geqq p^{\prime}$. Thus $q \geqq \bigcup\left(H_{Q}-p\right)$. Since $q \geqq p$ it follows that $\mathrm{U}\left(H_{Q}-p\right) \geq p$. Thus $H_{Q}$ is independent and (1) holds. (3) follows immediately from (2). 
According to Theorem 3.1 each element $a$ has at least one irredundant decomposition into irreducibles. For modular lattices a much stronger existence theorem holds.

THEOREM 4.3. Let a be an element of a compactly generated, atomic, modular lattice and let $a=\cap Q$ be an irredundant decomposition of $a$ into irreducibles. Then if $J$ is an independent subset of $P_{a}$ such that $J \supseteq H_{Q}$, there exists an irredundant decomposition $a=\cap Q^{\prime}$ such that $H_{Q^{\prime}}=J$.

Proof. By a trivial application of the Maximal Principle $J$ can be extended to a maximal independent set $M \subseteq P_{a}$. Let $J_{1}=J-H_{Q}$ and $M_{1}=M-J_{1}$. If we set $b=\bigcup J_{1}$ and $c=\cup M_{1}$, then by Lemma 3.6, $b \cup c=\cup M=u_{a}$ and by Lemma 3.7, $b \cap c=a$. For each $p \in H_{Q}$, let $q_{p}$ be the unique element of $Q$ such that $\bigcap\left(Q-q_{p}\right) \geqq p$. Then $q_{p} \geqq p$ and hence $q_{p} \cup p>q_{p}$. Since $q_{p}$ is irreducible, $q_{p} \cup p_{1}=q_{p} \cup p$ for any other $p_{1} \in M_{1}$ such that $q_{p} \geq p_{1}$. Thus $q_{p} \cup c=q_{p} \cup \cup M_{1}$ $=q_{p} \cup p>q_{p}$. By modularity we have $c>c \cap q_{p}$. Now $b \cup\left(c \cap q_{p}\right) \neq u_{a}$, since otherwise $c=c \cap u_{a}=c \cap\left(b \cup\left(c \cap q_{p}\right)\right)=(c \cap b) \cup\left(c \cap q_{p}\right)=c \cap q_{p}$ contrary to $c>c \cap q_{p}$. Thus $u_{a}=b \cup c>b \cup\left(c \cap q_{p}\right)$. Let us set $s_{p}=b \cup\left(c \cap q_{p}\right)$ so that $s_{p}$ is a maximal element of $u_{a} / a$ for each $p \in H_{Q}$.

Now for each $p \in J_{1}$ let us set $s_{p}=\bigcup\left(J_{1}-p\right) \cup c$. Clearly $u_{a}>s_{p}$ for each $p \in J_{1}$. Furthermore, since $J_{1}$ is an independent subset of $P_{a}$ it follows from Lemma 3.7 that $\bigcap_{p} \cup\left(J_{1}-p\right)=a$. Since $L$ is modular, the mapping $x \rightarrow x \cup c$ maps the quotient $b / a=b / b \cap c$ isomorphically onto $b \cup c / c=u_{a} / c$. Hence $\bigcap_{p \in J_{1}} s_{p}=c$. Thus

$$
\begin{aligned}
\bigcap_{J}= & \bigcap_{J_{1}} s_{p} \cap \bigcap_{H_{Q}} S_{p}=c \cap \bigcap_{H_{Q}}\left(b \cup\left(c \cap q_{p}\right)\right)=\bigcap_{H_{Q}}\left(c \cap\left(b \cup\left(c \cap q_{p}\right)\right)\right) \\
& =\bigcap_{H_{Q}}\left((c \cap b) \cup\left(c \cap q_{p}\right)\right)=\bigcap_{H_{Q}}\left(c \cap q_{p}\right)=c \cap \cap Q=a .
\end{aligned}
$$

It follows immediately from the definition that if $p \in J_{1}$, then $s_{p} \geqq c \geqq \cup H_{Q}$ and if $p \in H_{Q}$, then $s_{p} \geqq b \geqq \cup J_{1}$. On the other hand, if $p \in J_{1}$, then $s_{p} \geqq U\left(J_{1}-p\right)$ and if $p \in H_{Q}$, then $s_{p} \geqq c \cap q_{p} \geqq \cup\left(H_{Q}-p\right)$. Thus for each $p \in J$ we have $s_{p^{\prime}} \geqq p$ all $p^{\prime} \neq p$ and hence $\bigcap\left\{s_{p^{\prime}} \mid p^{\prime} \neq p, p \in J\right\} \geqq p$. By Lemma 2.5 for each $p \in J$ there exists a completely meet irreducible element $q_{p}^{\prime}$ such that $q_{p}^{\prime} \geqq s_{p}$ and $q_{p}^{\prime} u_{a}$. Let $Q^{\prime}=\left\{q_{p}^{\prime} \mid p \in J\right\}$. Then $u_{a} \cap q_{p}^{\prime}=s_{p}$ and hence by Lemma 3.2, $\cap Q^{\prime}=a$ is an irredundant decomposition of $a$. Clearly $H_{Q^{\prime}}=J$.

For finite irreducible decompositions it is easily verified that $U_{q} \cap(Q-q)$ $\geqq u_{a}$. This property no longer holds for general decompositions. In fact, we shall show that $U_{Q} \cap(Q-q) \cap u_{a}=h_{Q}$. A preliminary lemma is needed.

Lemma 4.4. Let $a \in L$ and let $a=\cap Q$ be an irredundant decomposition of $a$ into irreducibles. Furthermore let $Q^{\prime}=\left\{q_{1}, \cdots, q_{n}\right\}$ be a finite subset of $Q$ such that $\cap\left(Q-q_{i}\right) \geqq p_{i}>a$, for each $i$. Then $\cap\left(Q-Q^{\prime}\right) \geqq p$ implies $p_{1} \cup \ldots \cup p_{n} \geqq p$ for each $p \in P_{a}$.

For $n=1$, the lemma follows immediately from Lemma 4.3. Now suppose 
that the lemma holds for $n=k-1$ and let $\cap\left(Q-\left\{q_{1}, \cdots, q_{k}\right\}\right) \geqq p>a$. If $q_{k} \geqq p$, then $\cap\left(Q-\left\{q_{1}, \cdots, q_{k-1}\right\}\right) \geqq p$ and hence by the induction hypothesis $p_{1} \cup \ldots \cup p_{k} \geqq p_{1} \cup \ldots \cup p_{k-1} \geqq p$. If $q_{k} \geqq p$, then since $q_{k} \geqq p_{k}$ we have $q_{k} \cap\left(p \cup p_{k}\right)>a$ by Lemma 4.2 . Now $\cap\left(Q-\left\{q_{1}, \cdots, q_{k-1}\right\}\right) \geqq q_{k} \geqq q_{k} \cap\left(p \cup p_{k}\right)$ and by the induction hypothesis we have $p_{1} \cup \ldots \cup \cup_{p_{k-1}} \geqq q_{k} \cap\left(p \cup_{p_{k}}\right)$. Then

$p_{1} \cup \ldots \cup p_{k} \geqq p_{k} \cup\left(q_{k} \cap\left(p \cup p_{k}\right)\right)=\left(p_{k} \cup q_{k}\right) \cap\left(p \cup p_{k}\right)$

$$
=\left(p \cup q_{k}\right) \cap\left(p \cup p_{k}\right) \geqq p .
$$

The lemma follows by induction.

THEOREM 4.4. If $a \in L$ and $a=\cap Q$ is an irredundant decomposition of $a$, then $\mathrm{U}_{q} \cap(Q-q) \cap u_{a}=h_{Q}$.

Proof. Let $\bigcup_{q} \cap(Q-q) \geqq p>a$. Then since $p$ is compact in $u_{a} / a$, there exists a finite subset $\left\{q_{1}, \cdots, q_{n}\right\}$ of $Q$ such that $U_{i} \cap\left(Q-q_{i}\right) \geqq p$. Thus $\cap\left(Q-\left\{q_{1}, \cdots, q_{n}\right\}\right) \geqq \cup_{i} \cap\left(Q-q_{i}\right) \geqq p$. Let $\cap\left(Q-q_{i}\right) \geqq p_{i}>a$. Then $p_{i} \in H_{Q}$ and by Lemma 4.4, $p_{1} \cup \ldots \cup p_{n} \geqq p$. Hence $h_{Q}=\cup H_{Q} \geqq p$ all $p \leqq \cup_{Q} \cap(Q-q)$. Since $u_{a} / a$ is a point lattice we have $h_{Q} \geqq U_{q} \cap(Q-q) \cap u_{a}$. But $U_{q} \cap(Q-q)$ $\cap u_{a} \geqq h_{Q}$ trivially.

In the theorems which follow it will be shown that the replacement properties of irredundant decompositions are determined by the order properties of the elements $h_{Q}$.

THEOREM 4.5. Let $a=\cap Q$ be an irredundant decomposition of $a \in L$ and let $a=\cap Q^{\prime}$ where $Q^{\prime}$ is obtained from $Q$ by replacing $q \in Q$ by an irreducible $q^{\prime}$. Then $h_{Q^{\prime}}=h_{Q}$.

Proof. According to Theorem 4.2 the decomposition $a=\cap Q^{\prime}$ is irredundant. Let $p$ be the unique element of $H_{Q}$ such that $\cap(Q-q) \geqq p$. Now for each $p^{*} \in H_{Q}-p$ let $q^{*}$ be the unique element of $Q$ such that $\cap\left(Q-q^{*}\right) \geqq p^{*}$ and let $p^{*}$ be the unique element of $H_{Q^{\prime}}$ such that $\cap\left(Q^{\prime}-q^{*}\right) \geqq p^{*}$. Clearly the correspondence $p^{*} \rightarrow p^{*}$ is a one-to-one mapping of $H_{Q}-p$ onto $H_{Q^{\prime}}-p$.

Now let $p^{*} \in H_{Q}-p$ be such that $p^{*} \neq p^{*}$ and let $q^{*}$ be the unique element of $Q$ for which $\cap\left(Q-q^{*}\right) \geqq p^{*}$. Then $q^{\prime} \geq p^{*}$ since otherwise $\cap\left(Q^{\prime}-q^{*}\right)$ $=\cap\left(Q-\left\{q, q^{*}\right\}\right) \cap q^{\prime} \geqq p^{*}$ and hence $p^{*}=\bar{p}^{*}$ contrary to assumption. Also $q^{\prime} \geq p$ and hence by Lemma 4.2, $q^{\prime} \cap\left(p \cup p^{*}\right)>a$. But then $\cap\left(Q^{\prime}-q^{*}\right)$ $=\cap\left(Q-\left\{q, q^{*}\right\}\right) \cap q^{\prime} \geqq q^{\prime} \cap\left(p \cup p^{*}\right)$ and thus $p^{*}=q^{\prime} \cap\left(p \cup p^{*}\right)$. It follows that $p \cup p^{*}=p \cup\left(q^{\prime} \cap\left(p \cup p^{*}\right)\right)=\left(p \cup q^{\prime}\right) \cap\left(p \cup p^{*}\right)=p \cup p^{*}$. But $p \cup p^{*}=p \cup p^{*}$ holds trivially if $p^{*}=p^{*}$. Thus for all $p^{*} \in H_{Q}-p$ we have $p \cup p^{*}=p \cup p^{*}$. Thus

$$
h_{Q^{\prime}}=\bigcup H_{Q^{\prime}}=\bigcup_{p^{*}}\left(p \cup p^{*}\right)=\bigcup_{p^{*}}\left(p \cup p^{*}\right)=\bigcup H_{Q}=h_{Q}
$$

This completes the proof of the theorem.

From Lemma 3.6 we get the following corollary to Theorem 4.5. 
CoROllary. Under the hypotheses of Theorem 4.5 , if $H_{Q}$ is a maximal independent set of $P_{a}$, then $H_{Q^{\prime}}$ is also a maximal independent set of $P_{a}$.

If the ascending chain condition is satisfied, then $h_{Q}=u_{a}$ for every irredundant decomposition $a=\cap Q$. Thus it is not surprising that an additional hypothesis is required for a simultaneous replacement theorem in the more general case.

THEOREM 4.6. If $a$ is an element of a compactly generated, atomic, modular, lattice and $a=\cap Q=\cap Q^{\prime}$ are two irredundant decompositions of $a$ with $h_{Q} \geqq h_{Q^{\prime}}$, then for each $q^{\prime} \in Q^{\prime}$ there exists $q \in Q$ such that $a=\cap(Q-q) \cap q^{\prime}=\cap\left(Q^{\prime}-q^{\prime}\right) \cap q$.

Proof. Suppose that the irredundant decompositions $a=\cap Q=\cap Q^{\prime}$ are such that $h_{Q} \geqq h_{Q^{\prime}}$, and that $q^{\prime} \in Q^{\prime}$. Let $p^{\prime} \in H_{Q^{\prime}}$ be such that $\cap\left(Q^{\prime}-q^{\prime}\right) \geqq p^{\prime}$. Since $\cup H_{Q} \geqq \cup H_{Q^{\prime}} \geqq p^{\prime}$, there exists a finite subset $\left\{p_{1}, \cdots, p_{n}\right\} \subseteq K_{Q}$ such that $p_{1} \cup \cdots \cup p_{n} \geqq p^{\prime}$. We may assume that $\left\{p_{1}, \cdots, p_{n}\right\}$ is a minimal such subset. Then if $q_{i} \in Q$ is such that $\cap\left(Q-q_{i}\right) \geqq p_{i}(i=1, \cdots, n)$, $\cap\left(Q-\left\{q_{1}, \cdots, q_{n}\right\}\right) \geqq p_{1} \cup \ldots \cup p_{n} \geqq p^{\prime}$. Moreover, if for some $i, q_{i} \geqq p^{\prime}$, then $\cap\left(Q-\left\{q_{1}, \cdots, q_{i-1}, q_{i+1}, \cdots, q_{n}\right\}\right) \geqq p^{\prime}$, and hence it follows from Lemma 4.4 that $p_{1} \cup \ldots \cup p_{i-1} \cup p_{i+1} \cup \ldots \cup p_{n} \geqq p^{\prime}$, contrary to the minimality of $\left\{p_{1}, \cdots, p_{n}\right\}$. Thus for each $i=1, \cdots, n, q_{i} \geq p^{\prime}$. Now $q^{\prime} \geq p^{\prime}$, and hence there must exist some $p_{j} \in\left\{p_{1}, \cdots, p_{n}\right\}$ such that $q^{\prime} \geq p_{j}$. Suppose $\cap\left(Q-q_{j}\right) \cap q^{\prime}>a$. Then since $L$ is atomic, $p \in P_{a}$ exists such that $\cap\left(Q-q_{j}\right) \cap q^{\prime} \geqq p$. But then $\cap\left(Q-q_{j}\right) \geqq p, p_{j}$, contradicting Lemma 4.3. Hence $\cap\left(Q-q_{j}\right) \cap q^{\prime}=a$. It is also true that $q_{j} \geq p^{\prime}$, and hence by a similar argument, $\cap\left(Q^{\prime}-q^{\prime}\right) \cap q_{j}=a$. The irredundancy of the decompositions $a=\cap\left(Q-q_{j}\right) \cap q^{\prime}$ $=\bigcap\left(Q^{\prime}-q^{\prime}\right) \cap q_{j}$ follows from Theorem 4.1.

CoROllary. Let $a \in L$ and $a=\cap Q$ be an irredundant decomposition of $a$. Then for each irredundant decomposition $a=\cap Q^{\prime}$ and each $q^{\prime} \in Q^{\prime}$, there exists $q \in Q$ such that $a=\bigcap(Q-q) \cap q^{\prime}$, if and only if $h_{Q}=u_{a}$.

Proof. If $h_{Q}=u_{a}$, then for any irredundant decomposition $a=\cap Q^{\prime}, h_{Q}$ $=u_{a} \geqq h_{Q^{\prime}}$, and hence Theorem 4.6 holds for the decompositions $a=\cap Q$ $=\cap Q^{\prime}$. Suppose $h_{Q}<u_{a}$. Then $H_{Q}$ is not a maximal independent subset of $P_{a}$, and hence there exists an independent subset $J \subseteq P_{a}$ such that $J \supset H_{Q}$. By Theorem 4.2, there exists an irredundant decomposition $a=\cap Q^{\prime}$ such that $H_{Q^{\prime}}=J$. Let $p \in H_{Q^{\prime}}-H_{Q}$, and let $q^{\prime} \in Q^{\prime}$ be such that $\cap\left(Q^{\prime}-q^{\prime}\right) \geqq p$. Then $q^{\prime} \geqq \cup H_{Q}$, and hence $\cap(Q-q) \cap q^{\prime}>a$ for all $q \in Q$. Thus $q^{\prime}$ can replace none of the irreducibles in the decomposition $a=\cap Q$.

The replacement property of Theorem 4.2 can be considerably sharpened. In order to simplify the statement of the theorem we shall introduce the notion of $Q$-equivalence.

Let $a=\cap Q$ be a decomposition of $a$, and let $S \subseteq Q$. A set $T$ of completely irreducible elements of $L$ is said to be $Q$-equivalent to $S$ if there is a one-one mapping $\phi$ of $S$ onto $T$ such that for each $q \in Q, a=\cap(Q-q) \cap \phi(q)$. 
THEOREM 4.7. If a is an element of a compactly generated, atomic, modular lattice and $a=\cap Q=\cap Q^{\prime}$ are two irredundant decompositions of $a$, then for each finite subset $S \subseteq Q$ there exists a subset $S^{\prime} \subseteq Q^{\prime}$ such that $S^{\prime}$ is $Q$-equivalent to $S$. If $h_{Q} \geqq h_{Q^{\prime}}$, then for each finite subset $S^{\prime} \subseteq Q^{\prime}$ there is a subset $S \subseteq Q$ such that $S^{\prime}$ is Q-equivalent to $S$.

Proof. For $n=1$, the first statement of this theorem is just that of Theorem 4.1. Let $\left\{q_{1}, \cdots, q_{n}\right\}$ be a finite subset of $Q$. For each $i=1, \cdots, n$, let $S_{i}=\left\{q^{\prime} \in Q \mid a=\cap\left(Q-q_{i}\right) \cap q^{\prime}\right\}$. Suppose for some $k$-element subset $\left\{q_{i_{1}}, \cdots, q_{i_{k}}\right\} \subseteq\left\{q_{1}, \cdots, q_{n}\right\}, k \leqq n, S_{i_{1}} \vee \cdots \vee S_{i_{k}}$ contains $m<k$ elements, say $q_{1}^{\prime}, \cdots, q_{m}^{\prime}$. Let $p_{i_{j}} \in H_{Q}$ be such that $\bigcap\left(Q-q_{i_{j}}\right) \geqq p_{i_{j}}(j=1, \cdots, k)$, and let $p_{j}^{\prime} \in H_{Q^{\prime}}$ be such that $\cap\left(Q^{\prime}-q_{j}^{\prime}\right) \geqq p_{j}^{\prime}(j=1, \cdots, m)$. Then for each $q^{\prime} \in Q^{\prime}-\left\{q_{1}^{\prime}, \cdots, q_{m}^{\prime}\right\}, \cap\left(Q-q_{i}\right) \cap q^{\prime}>a$, and hence, since $\cap\left(Q-q_{i}\right)$ contains only one element of $P_{a}, q^{\prime} \geqq p_{i}$ for each $i=1, \cdots, k$. Thus

$$
\cap\left(Q^{\prime}-\left\{q_{1}^{\prime}, \cdots, q_{m}^{\prime}\right\}\right) \geqq p_{1} \cup \cdots \cup p_{k},
$$

and it follows from Lemma 4.4 that $p_{1}^{\prime} \cup \ldots \cup p_{m}^{\prime} \geqq p_{1} \cup \ldots \cup p_{k}$. But this is impossible, since the dimension $m$ of the quotient $p_{1}^{\prime} \cup \ldots \cup p_{m}^{\prime} / a$ is less than the dimension $k$ of the quotient $p_{1} \cup \ldots \cup p_{k} / a$. Hence for each $k$-element subset $\left\{q_{i_{1}}, \cdots, q_{i_{k}}\right\} \subseteq\left\{q_{1}, \cdots, q_{n}\right\}, S_{i_{1}} \vee \cdots \vee S_{i_{k}}$ contains at least $k$ distinct elements. It now follows from P. Hall's theorem on representatives of subsets that there are $n$ distinct elements $q_{1}^{\prime}, \cdots, q_{n}^{\prime} \in Q^{\prime}$ such that $q_{i}^{\prime} \in S_{i}(i=1, \cdots, n)$, completing the proof of the first part.

Now let $h_{Q} \geqq h_{Q^{\prime}}$. Let $\left\{q_{1}^{\prime}, \cdots, q_{n}^{\prime}\right\}$ be a finite $n$-element subset of $Q^{\prime}$, and let $T_{i}=\left\{q \in Q \mid a=\cap(Q-q) \cap q_{i}^{\prime}\right\}$. In view of the preceding paragraph, to prove the second part of the theorem it suffices to show that $T_{1} \vee \cdots \vee T_{n}$ contains at least $n$ distinct elements. Suppose $T_{1} \vee \cdots \vee T_{n}$ contains $m<n$ elements, say $q_{1}, \cdots, q_{m}$. For each $i=1, \cdots, m$, let $p_{i} \in H_{Q}$ be such that $\cap\left(Q-q_{i}\right) \geqq p_{i}$. Then if $p \in H_{Q}-\left\{p_{1}, \cdots, p_{m}\right\}, q_{1}^{\prime} \geqq p$ for each $i=1, \cdots, n$, and hence $q_{1}^{\prime} \cap \cdots \cap q_{n}^{\prime} \geqq \mathrm{U}\left(H_{Q}-\left\{p_{1}, \cdots, p_{m}\right\}\right)$. Since $h_{Q} \geqq U H_{Q^{\prime}}$, and since for each $i, q_{i}^{\prime}$ is covered by only one element, $h_{Q} \cup q_{i}^{\prime}>q_{i}^{\prime}$ and hence $h_{Q}>h_{Q} \cap q_{i}^{\prime}$. Furthermore if $k<n$, then $\left(h_{Q} \cap q_{1}^{\prime} \cap \ldots \cap q_{k}^{\prime}\right) \cup q_{k+1}^{\prime}>q_{k+1}^{\prime}$, and hence $h_{Q} \cap q_{1}^{\prime} \cap \cdots \cap q_{k}^{\prime}>h_{Q} \cap q_{1}^{\prime} \cap \cdots \cap q_{k+1}^{\prime}$. Thus the quotient sublattice $h_{Q} / h_{Q} \cap q_{1}^{\prime} \cap \cdots \cap q_{n}^{\prime}$ is of dimension $n$. But now we have a contradiction, since the quotient $h_{Q} / \mathrm{U}\left(H_{Q}-\left\{p_{1}, \cdots, p_{m}\right\}\right)$ is of dimension $m<n$. Hence $T_{1} \vee \cdots \vee T_{n}$ contains at least $n$ distinct elements, and the proof is complete.

COROLlary. If $a$ is an element of a compactly generated, atomic, modular lattice and $a=\cap Q=\cap Q^{\prime}$ are two irredundant decompositions of $a$ with $h_{Q} \leqq h_{Q^{\prime}}$, then there exists a subset $S^{\prime} \subseteq Q^{\prime}$ such that $S^{\prime}$ is Q-equivalent to $Q$.

Proof. For each $q \in Q$, let $S_{q}=\left\{q^{\prime} \in Q^{\prime} \mid a=\cap(Q-q) \cap q^{\prime}\right\}$. Then from Theorem 4.7 it follows that for any finite subset $\left\{q_{1}, \cdots, q_{n}\right\} \subseteq Q, S_{q_{1}} \vee \cdots$ $\vee S_{q_{n}}$ contains at least $n$ distinct elements. Now suppose $q \in Q$ and $p \in H_{Q}$ 
is such that $\cap(Q-q) \geqq q$. Since $h_{Q} \leqq h_{Q^{\prime}}, p \leqq \cup H_{Q}$, and hence $p \leqq p_{1}^{\prime} \cup \ldots \cup p_{p_{k}^{\prime}}$ for some finite subset $\left\{p_{1}^{\prime}, \cdots, p_{k}^{\prime}\right\} \subseteq Q^{\prime}$. If $q_{i}^{\prime}$ is such that $\cap\left(Q^{\prime}-q_{i}^{\prime}\right)$ $\geqq p_{i}^{\prime}(i=1, \cdots, k)$, then $\cap\left(Q^{\prime}-\left\{q_{1}^{\prime}, \cdots, q_{k}^{\prime}\right\}\right) \geqq p$, and thus $\left\{q_{1}^{\prime}, \cdots, q_{k}^{\prime}\right\}$ $\supseteq S_{q}$. Hence for each $q \in Q, S_{q}$ is finite. The corollary now follows from the Marshall Hall theorem (Hall [6]) on representatives of subsets.

Corollary. If $a=\cap Q=\cap Q^{\prime}$ are two irredundant decompositions of $a \in L$ such that $h_{Q}=h_{Q^{\prime}}$, then $Q$ and $Q^{\prime}$ have the same cardinality.

We conclude this section with an example of a compactly generated, atomic, modular lattice in which the null element has two irredundant decompositions into irreducibles of different cardinalities.

For each integer $i$, let $A_{i}$ be a group isomorphic with the additive group of integers modulo a fixed prime $p$, and let $G$ be the complete direct sum of the groups $A_{i}$, that is, the set of all functions $f$ on the integers such that $f(i) \in A_{i}$, with addition defined componentwise. Then $G$ is an (additive) abelian group every element of which has order $p$. Let $L$ be the lattice of subgroups of $G$. $L$ is then compactly generated and modular, and since every element of $G$ has finite order, $L$ is also atomic. For each $i$, let $Q_{i}$ be that subgroup of $G$ consisting of all functions $f \in G$ for which $f(i)$ is the zero element of $A_{i}$. Then $G / Q_{i}$ is isomorphic with $A_{i}$ and hence $Q_{i}$ is a maximal subgroup of $G$. Thus $Q_{i}$ is a completely irreducible element of $L$ for each $i$. Since $G$ is the complete direct sum of the $A_{i}$, it follows that $0=\bigcap_{i} Q_{i}$ (0 denoting the zero subgroup of $G)$. Moreover, for each $i, Q_{i} \cup\left(\bigcap_{j \neq i} Q_{j}\right)=G$, and hence the decomposition $0=\bigcap_{i} Q_{i}$ is irredundant. Now $G$ can be considered as a vector space over the field of integers modulo $p$, and accordingly, $G$ has a basis $\left\{f_{\alpha}\right\}$. Since $G$ has cardinality $2^{\mathbb{N}_{0}}$, the number of $f_{\alpha}$ must also be $2^{\aleph_{0}}$. For each index $\alpha$, let $Q_{\alpha}^{\prime}$ be that subgroup of $G$ generated by the set $\left\{f_{\beta} \mid \beta \neq \alpha\right\}$. Then each $Q_{\alpha}^{\prime}$ is a maximal subgroup of $G$, and just as above, it follows that $0=\bigcap_{\alpha} Q_{\alpha}^{\prime}$ is an irredundant decomposition of 0 . Thus $0 \in L$ has two irredundant decompositions with different cardinalities.

5. Distributive lattices. Under the hypothesis of the ascending chain condition, each element of a distributive lattice has a unique irredundant decomposition into irreducibles (Birkhoff $[1$, p. 142]). This decomposition is necessarily finite. In this section, we shall show that there exists a unique (though possibly infinite) irredundant decomposition for each element of a compactly generated atomic distributive lattice.

We begin with a theorem concerning irreducibles in semimodular lattices.

THEOREM 5.1. Let a be an element of a semimodular, compactly generated, atomic lattice. Then an irreducible $q$ appears in every irredundant decomposition of $a$ if and only if there exists $p>a$ with $p \leq q$ such that $p \cap(x \cup y)=(p \cap x)$ $\cup(p \cap y)$ for all $x, y \geqq a$.

Proof. Suppose such a $p$ exists. Let $q^{\prime}$ be an irreducible with $q^{\prime} \geq p$. Then $p \cap q=p \cap q^{\prime}=a$, and hence $a=(p \cap q) \cup\left(p \cap q^{\prime}\right)=p \cap\left(q \cup q^{\prime}\right)$. Now if $q \neq q^{\prime}$, 
then $q \cup q^{\prime} \geqq u_{a} \geqq p$ contrary to $p \geq q \cup q^{\prime}$. Thus $q=q^{\prime}$, and $q$ is the only irreducible not containing $p$. Hence $q$ appears in every decomposition of $a$.

Suppose $q$ appears in every irredundant decomposition of $a$. Let $p$ be an element covering $a$ such that $q \geqq p$. Let $x, y \geqq a$, and suppose $x \cap p=y \cap p=a$. By Lemma 2.5 an irreducible element $q_{x}$ exists such that $q_{x} \geq p$ and $q_{x} \geqq x$. Similarly an irreducible element $q_{\nu}$ exists. Thus by Theorem 3.1 there are irredundant decompositions of $a$ which contain $q_{x}$ and $q_{y}$, respectively. But $q$ appears in every irredundant decomposition of $a$ and $q \geq p$, whence it follows that $q=q_{x}=q_{y}$. Hence $q \geqq x \cup y$, so that $x \cup y \geqq p$. Thus $p \cap(x \cup y)=(p \cap x)$ $\cup(p \cap y)$, and the theorem follows.

Combining Theorems 3.1 and 5.1 we have the following theorem.

THEOREM 5.2. Every element of a compactly generated, atomic, distributive lattice has a unique irredundant decomposition into irreducibles.

For distributive lattices satisfying the ascending chain condition it is easy to show that $q^{\prime} \geqq a$ implies $q^{\prime} \geqq q$ where $q$ belongs to the unique irredundant decomposition of $a$. This property does not generalize to compactly generated, atomic, distributive lattices. Consider, for example, the collection of all subsets $S$ of the set $I$ of positive integers such that either $S \subseteq I-\{1\}$ or $I-S$ is finite. It can be easily verified that this collection is closed under arbitrary union and finite intersection and thus is a complete distributive lattice. The compact elements of this lattice are the finite subsets of $I-\{1\}$ and the sets $S$ such that $1 \in S$. These sets clearly generate the lattice under arbitrary union and hence the lattice is compactly generated. The lattice is obviously atomic. Now the unique irredundant decomposition of the null set is $\varnothing=\cap_{n=2}^{\infty} Q_{n}$ where $Q_{n}=I-\{n\}$. Note that this lattice meet is not set intersection since $\Lambda_{n=2}^{\infty} Q_{n}=\{1\}$. On the other hand, $Q_{1}=I-\{1\}$ is an irreducible containing $\varnothing$ such that $Q_{1} \supseteq Q_{n}$ for every $n \neq 1$.

6. Unique decompositions. In the previous section we have shown that each element of a distributive, compactly generated, atomic lattice has a unique irredundant decomposition into irreducibles. This section will be devoted to a characterization of lattices having unique irredundant decompositions into irreducibles. The characterization will be analogous to the finite dimensional case (Dilworth [2]), though quite different techniques of proof are required. We begin with the definition of the relevant concepts.

Definition 6.1. An atomic lattice $L$ is locally distributive (locally modular) if $u_{a} / a$ is distributive (modular) for each $a \in L$.

Clearly a locally distributive or locally modular lattice is weakly semimodular. Hence from Lemma 3.3 we have

Lemma 6.1. A locally distributive or locally modular, compactly generated, atomic lattice is semimodular.

We first show that for compactly generated atomic lattices, unique irredundant decompositions imply that the lattice is semimodular. 
Lemma 6.2. Let $L$ be a compactly generated atomic lattice. Then if every element of $L$ has a unique irredundant decomposition into irreducibles, $L$ is semimodular.

For let $p>a, b \geqq a$, and $b$. Suppose $p \cup b$ does not cover $b$. Then $x$ exists such that $p \cup b>x>b$. By Lemma 2.5 there exists an irreducible $q$ such that $q \geqq b$ and $q \geqq x$. Similarly there exists an irreducible $q^{\prime}$ such that $q^{\prime} \geqq x$ and $q^{\prime} \geq p \cup b$. Now $a=p \cap q=p \cap q^{\prime}$. By the Maximal Principal elements $m$ and $m^{\prime}$ exist which are maximal such that $m \cap q=a, m \geqq p$, and $m^{\prime} \cap q^{\prime}=a, m^{\prime} \geqq p$. By assumption $m$ and $m^{\prime}$ have irredundant decompositions $m=\cap M$ and $m^{\prime}=\cap M^{\prime}$. Clearly $q \notin M^{\prime}$ and $q^{\prime} \notin M$. However, $a=q \cap \cap M$ $=q^{\prime} \cap \cap M^{\prime}$ are two different irredundant decompositions of $a$, contrary to uniqueness. Thus $p \cup b>b$, and $L$ is semimodular.

The next lemma is a direct consequence of Theorem 5.1 and Lemma 3.7 (and its corollary).

Lemma 6.3. Let $L$ be a semimodular, compactly generated, atomic lattice. If $a \in L$ has a unique irredundant decomposition into irreducibles, then $u_{a} / a$ is distributive.

It should be noted that in any compactly generated atomic lattice, $u_{a} / a$ will be a complete atomic, Boolean algebra if it is distributive. We now turn to the converse of the above lemma.

LEMma 6.4. Let $L$ be a locally distributive, compactly generated, atomic lattice. Then each element of $L$ has a unique irredundant decomposition into irreducibles.

From Lemma 6.1 it follows that $L$ is semimodular. We will show first that if $p>a$ and if $q_{1}$ and $q_{2}$ are irreducibles such that $q_{1}, q_{2} \geqq a$ and $q_{1}, q_{2} \geq p$, then $q_{1}=q_{2}$. If $q_{1} \neq q_{2}$, then $q_{1} \geqq q_{2}$ since otherwise $q_{1} \geqq q_{2} \cup p \geqq p$ contrary to $q_{1} \geqq p$. Thus $q_{2}>q_{1} \cap q_{2}$ and hence there exists $b_{2}$ such that $q_{2} \geqq b_{2}>q_{1} \cap q_{2}$. Since $q_{2} \geq p$ we have $b_{2} \geqq p$. Now let $S=\left\{s \mid q_{1} \geqq s \geqq q_{1} \cap q_{2}, s \cup b_{2} \geqq p\right\}$. $S$ is nonempty since $q_{1} \cap q_{2} \in S$. Furthermore, since $p$ is compact in $q_{1} / a, S$ is inductive. Let $s^{*}$ be a maximal element of $S$. If $q_{1}>s^{*}$, then there exists $w_{1}$ such that $q_{1} \geqq w_{1}>s^{*}$. Since $s^{*} p$ and $s^{*} \geqq a$ we have $w_{2}=s^{*} \cup p>s^{*}$ by semimodularity. Also $s^{*} \geqq b_{2}$, since otherwise $q_{1} \geqq s^{*} \geqq b_{2}$ and hence $q_{1} \cap q_{2} \geqq b_{2}$ contrary to $b_{2}>q_{1} \cap q_{2}$. Since $s^{*} \geqq q_{1} \cap q_{2}$ we have $w_{3}=s^{*} \cup b_{2}>s^{*}$ by semimodularity. Now $w_{1} \neq w_{2}$ since $q_{1} \geq p ; w_{1} \neq w_{3}$ since $q_{1} \geq b_{2}$; and $w_{2} \neq w_{3}$ since $s^{*} \cup b_{2} \geq p$. By local distributivity $w_{1} \cup w_{3} \geq w_{2}$. But then $w_{1} \cup b_{2}=w_{1} \cup s^{*} \cup b_{2}=w_{1} \cup w_{3} \geq p$ and hence $w_{1} \in S$ contrary to the maximal property of $s^{*}$. Thus we must have $q_{1}=s^{*}$ and hence $q_{1} \cup b_{2} \geq p$. But then $q_{1} \cup b_{2}=q_{1}$, since otherwise $q_{1} \cup b_{2}>q_{1}$, $q_{1} \cup p>q_{1}$ and $q_{1} \cup b_{2} \neq q_{1} \cup p$ contrary to the irreducibility of $q_{1}$. Thus $q_{1} \geqq b_{2}$, and hence $q_{1} \cap q_{2} \geqq b_{2}$ contrary to $b_{2}>q_{1} \cap q_{2}$. It follows that our original assumption is untenable and hence $q_{1}=q_{2}$.

Now let $q$ be irredundant in the decomposition $a=\cap Q$ and let $q \geqq \cap\left(Q^{\prime}-q^{\prime}\right)$ 
in the decomposition $a=\cap Q^{\prime}$. Then $q$ 主 $p$ some $p \in P_{a}$. Now if $q^{\prime \prime} \neq q$ all $q^{\prime \prime} \in Q^{\prime}$ we have $q^{\prime \prime} \geqq p$ all $q^{\prime \prime} \in Q^{\prime}$ by the result of the preceding paragraph. But then $a=\cap Q^{\prime} \geqq p$ contrary to $p>a$. Hence $q=q^{\prime \prime}$ some $q^{\prime \prime} \in Q^{\prime}$. But if $q^{\prime \prime} \neq q^{\prime}$ we have $q \geqq \bigcap\left(Q^{\prime}-q^{\prime}\right)$. Thus $q=q^{\prime}$ and $a$ has a unique decomposition into irreducibles. This completes the proof of the lemma.

Combining Lemmas 6.1-6.4 we have

TheOREM 6.1. A compactly generated, atomic lattice has unique irreducible decompositions if and only if it is locally distributive.

An examination of the proof of Lemma 6.4 shows that the only conditions required for the sufficiency argument are weak semimodularity and the fact that every three distinct elements covering an element of the lattice are independent. Thus we have the following corollary.

CoRollary. A compactly generated atomic lattice is locally distributive if and only if every three distinct covering elements generate a dense Boolean algebra of order eight.

A further characterization analogous to that of Theorem 1.1 of [2] is the following:

CoROllary. A semimodular, compactly generated, atomic lattice is locally distributive if and only if every modular sublattice is distributive.

If every modular sublattice is distributive then every three distinct covering elements must be independent and hence by the first corollary to Theorem 6.1 , the lattice is locally distributive.

Now let $L$ be locally distributive and let $M$ be a modular sublattice which is not distributive. We may clearly suppose that $M$ is the modular, nondistributive lattice of order five. Hence there exist elements $a, b, c, d, e$ such that $b \cup c=b \cup d=c \cup d=e$ and $b \cap c=b \cap d=c \cap d=a$. It now follows (see Lemma 7.2 below) that there exist two irredundant decompositions $a=\cap Q$ $=\cap Q^{\prime}$ such that for each $q \in Q$ either $q \geqq b$ or $q \geqq c$, and for each $q^{\prime} \in Q^{\prime}$ either $q^{\prime} \geqq b$ or $q^{\prime} \geqq d$. Let $q \in Q$ be such that $q \geqq b$. By Theorem $6.1, q \in Q^{\prime}$ and hence $q \geqq d$. But then $q \geqq c \cup d \geqq b$, a contradiction. Thus every modular sublattice of $L$ is distributive.

7. Locally modular lattices. The problem of the characterization of lattices in which the number of components in the irredundant decompositions of elements is unique is much more complex than the uniqueness problem treated in $\S 6$. In fact it is easy to give examples of nonsemimodular lattices in which the number of components is unique. Since a decomposition theory for lattices which are not semimodular has not yet been developed we will restrict our discussion to the semimodular case.

When a semimodular lattice satisfies the ascending chain condition the decompositions are finite and the basic result states that the number of com- 
ponents in the irredundant representations is unique if and only if $L$ is locally modular (Dilworth [4]). For infinite decompositions, the example given at the end of $\$ 4$ seems to indicate that questions concerning the invariance of the cardinality of decompositions are primarily set theoretic in nature. On the other hand, in the finite case, the invariance of the number of components is always obtained in terms of replacement properties and these properties are of a lattice theoretic character. Accordingly in this section we shall investigate the structure of semimodular, compactly generated, atomic lattices in which the fundamental replacement property of Theorem 4.2 holds. It will turn out that the lattices satisfying this replacement property will be precisely the locally modular lattices.

Definition 7.1. A lattice is said to have replaceable decompositions if for every element $a$ of the lattice, each irreducible in one irredundant decomposition of $a$ can be replaced by a suitable irreducible in any other irredundant decomposition of $a$.

The following lemma and its corollary show the sufficiency of local modularity for replaceability.

Lemma 7.1. Let $L$ be a locally modular, compactly generated, atomic lattice. Then $q \geqq a$ and $q \geq u_{a}$ imply $u_{a}>q \cap u_{a}$ for each irreducible $q$.

For suppose that $u_{a}>q \cap u_{a}$ does not hold. Then there exist two distinct elements $p_{1}$ and $p_{2}$ in $P_{a}$ such that $\left(q \cap u_{a}\right) \cap\left(p_{1} \cup p_{2}\right)=a$ and hence $q \cap\left(p_{1} \cup p_{2}\right)$ $=a$. Now let $X$ be a chain of elements $x$ such that $q \geqq x \geqq a, q \cap\left(x \cup p_{1} \cup p_{2}\right)=x$, $x \cup p_{1} \geqq p_{2}$. Then $q \geqq \bigcup X \geqq a$ and by continuity $\bigcup X=q \cap \bigcup_{x}\left(x \cup p_{1} \cup p_{2}\right)$ $=q \cap\left(\cup X \cup p_{1} \cup p_{2}\right)$. Also $\cup X \cup p_{1} \geq p_{2}$ since $p_{2}$ is compact in $u_{a} / a$. Thus $\cup X$ satisfies the condition on $x$ and since $a$ trivially satisfies the conditions, it follows from the Maximal Principle that there is a maximal element $m$ for which $q \geqq m \geqq a . q \cap\left(m \cup p_{1} \cup p_{2}\right)=m, m \cup p_{1} \geq p_{2}$. Now clearly $m \cup p_{1} \neq m \cup p_{2}$ and $m \cup p_{1}>m, m \cup p_{2}>m$. Thus $m=\left(m \cup p_{1}\right) \cap\left(m \cup p_{2}\right)$ and hence is reducible. It follows that $q>m$ and by atomicity we have $q \geqq m_{1}>m$. If $m_{1} \cup p_{1} \geqq p_{2}$, then $m_{1} \cup_{p_{1}}=m \cup p_{1} \cup p_{2}$ and $m=q \cap\left(m \cup_{p_{1}} \cup_{p_{2}}\right)=q \cap\left(m_{1} \cup_{p_{1}}\right) \geqq m_{1}$ contrary to $m_{1}>m$. Thus $m_{1} \cup_{p_{1}} \geqq p_{2}$. Since $q \geqq m_{1} \geqq a$, by the maximal property of $m$ we must have $q \cap\left(m_{1} \cup p_{1} \cup p_{2}\right)>m$. From $m_{1} \cup p_{1} \cup p_{2}>m_{1} \cup p_{1}>m_{1}$ we conclude that $m_{1} \cup p_{1} \cup p_{2}>q \cap\left(m_{1} \cup p_{1} \cup p_{2}\right)$. But $m_{1} \cup p_{1} \cup p_{2} \in u_{m} / m$ and since $u_{m} / m$ is modular by hypothesis, we have $m \cup_{p_{1}} \cup_{p_{2}}>q \cap\left(m \cup \cup_{1} \cup_{p_{2}}\right)$ $=m$. Hence $m \cup p_{1}=m \cup p_{1} \cup p_{2} \geqq p_{2}$ contrary to $m \cup p_{1} \geq p_{2}$. It follows from this final contradiction that $u_{a}>q \cap u_{a}$.

Corollary. A locally modular, compactly generated, atomic lattice has replaceable decompositions.

For, by Lemma 7.1, each irreducible containing $a$ is such that $q \cap u_{a}$ is an irreducible element of $u_{a} / a$. But by Theorem 4.2 decompositions in $u_{a} / a$ are replaceable. Hence by Lemma 3.2 decompositions in $L$ are replaceable. 
The necessity of local modularity is a considerably deeper result. We begin with two preliminary lemmas which will be needed later in the proof. The second lemma concerning finite dimensional semimodular lattices is of interest as a generalization of a well known classical theorem.

Lemma 7.2. Let $L$ be a compactly generated, atomic lattice in which each element has an irredundant decomposition into irreducibles. Then if $a=a_{1} \cap \ldots$ $\cap a_{n}$ is an irredundant finite representation of a, there exists an irredundant decomposition $a=\cap Q$ such that for each $q \in Q, q \geqq a_{i}$ for some $i$.

For since $L$ is continuous there exists a maximal element $m_{1} \geqq a_{1}$ such that $a=m_{1} \cap a_{2} \cap \cdots \cap a_{n}$. Similarly there exists a maximal element $m_{2} \geqq a_{2}$ such that $a=m_{1} \cap m_{2} \cap a_{3} \cap \cdots \cap a_{n}$. By induction we get $a=m_{1} \cap m_{2} \cap \cdots \cap m_{n}$ where $m_{i}$ is maximal such that $a=m_{1} \cap \cdots \cap m_{i} \cap a_{i+1} \cap \cdots \cap a_{n}$. Thus $m_{i}^{\prime}>m_{i}$ implies $m_{1} \cap \ldots \cap m_{i-1} \cap m_{i}^{\prime} \cap m_{i+1} \cap \ldots \cap m_{n}>a$. Now let $m_{i}=\cap Q_{i}$ be an irredundant decomposition of $m_{i}$ into irreducibles and let $Q=\vee_{i} Q_{i}$. Then $\cap Q=\bigcap_{i} \cap Q_{i}=\bigcap_{i} m_{i}=a$. Let $q_{i} \in Q_{i}$ then $\cap\left(Q_{i}-q_{i}\right)>m_{i}$. Hence $\cap\left(Q-q_{i}\right) \geqq m_{1} \cap \ldots \cap m_{i-1} \cap\left(Q_{i}-q_{i}\right) \cap m_{i+1} \cap \cdots \cap m_{n}>a$. Thus the decomposition $a=\cap Q$ is irredundant.

LeMma 7.3. Let $L$ be a finite dimensional semimodular lattice in which the unit element $u$ is a join of points. Let $B$ denote the set of elements of $L$ which are joins of points. Then if $u>s, s \geqq b$ imply $b>s \cap b$ for all $b \in B, L$ is modular.

Proof. Let us suppose that $L$ contains an element which is not a join of points. Then by finite dimensionality there exists a minimal element $b \in B$ such that $b / z$ contains an element $x$ which is not a join of points. Let $b>y \geqq x$. By the minimal property of $b, y$ is not a join of points. Let $b_{1}$ be the union of points in $y$. Then $b>y>b_{1}$. Since every element of $L$ distinct from $u$ is a meet of maximal elements, it follows that there exists $s$ such that $u>s$, $s \geqq b$ and $s \geqq y$. Thus $s \cap b=y$. Now let $S$ be a maximal independent set of points in $b_{1}$. Then $b_{1}=U S$ by Lemma 3.6. Let $S$ be extended to a maximal independent set $T$ of points in $b$. Then $b=\cup T . S-T$ clearly contains at least two distinct points since otherwise $b=U T>U S=b_{1}$ contrary to $b>y>b_{1}$. Let $p, q$ be distinct points of $S-T$. Then $s \geqq p, q$ since otherwise $y=b \cap s \geqq p, q$ and $b_{1}=\cup S \geqq p, q$, contrary to the independence of $T$. By the hypothesis of the lemma $p \cup q>s \cap(p \cup q)=s \cap b \cap(p \cup q)=y \cap(p \cup q)$. Thus $y \cap(p \cup q)$ is a point contained in $y$. Hence $b_{1} \geqq y \cap(b \cup q)$. But then $y \cap(p \cup q) \leqq y \cap b_{1}$ $\cap(p \cup q)=\cup(S) \cap(p \cup q)=z$ since $T$ is independent contrary to $y \cap(p \cup q)>z$. In view of this contradiction we conclude that every element of $L$ is a join of points. Now let $a \cup b>b$ for $a, b \in L$. Let $u>s, s \geqq b, s \geqq a \cup b$. Then $s \cap(a \cup b)$ $=b$. Clearly $s \geqq a$ and since $a$ is a join of points we have $a>s \cap a=s \cap(a \cup b)$ $\cap a=a \cap b$. Then $L$ is lower semimodular and since it is upper semimodular by hypothesis, it is modular. This completes the proof of the lemma.

It will now be shown that the modularity of $u_{a} / a$ follows from the replacement property for the irredundant decompositions of $a$. 
LEMMA 7.4. Let $L$ be a semimodular, compactly generated, atomic lattice such that the replacement property holds for the irredundant decompositions of $a$. Then $u_{a} / a$ is modular.

It will first be shown that $u_{a}>s, s \geqq b$, where $b$ is a join of elements of $P_{a}$ imply that $b>s \cap b$.

Let us suppose that this is not the case and hence that $u_{a}>s, s \geqq b$ while $b>s \cap b$ does not hold for some $b$ which is a join of elements of $P_{a}$. Now let $T$ be a maximal independent set of elements of $P_{a}$ contained in $s \cap b$. Extend $T$ to a maximal independent set $S$ of elements of $P_{a}$ contained in $b$. Then by Lemma 3.6, $\cup S=b$. Finally extend $S$ to a maximal independent set $P$ of points in $P_{a}$. For each $p \in P$ let $q_{p}$ be an irreducible such that $q_{p} \geqq U(P-p)$, $q_{p} \geq u_{a}$. Set $Q=\left\{q_{p} \mid p \in P\right\}$. Then by Lemma 3.7, $a=\cap Q$ is an irredundant decomposition of $a$ into irreducibles. Now consider the case when $s \cap b=a$. Then by Lemma 7.2 there exists an irredundant decomposition $a=\cap Q^{\prime}$ such that $q^{\prime} \geqq s$ or $q^{\prime} \geqq b$ for each $q^{\prime} \in Q^{\prime}$. Since $b \neq a$, there exists at least one $q^{\prime} \in Q^{\prime}$ such that $q^{\prime} \geqq s$. Suppose that there is another $q^{\prime \prime} \in Q^{\prime}$ such that $q^{\prime \prime} \geqq s$. Then $u_{a} \cap q^{\prime}=s=u_{a} \cap q^{\prime \prime}$ and hence $u_{a} \cap \cap\left(Q^{\prime}-q^{\prime}\right)=u_{a} \cap q^{\prime \prime} \cap \cap\left(Q^{\prime}-q^{\prime}\right)$ $=u_{a} \cap q^{\prime} \cap \cap\left(Q^{\prime}-q^{\prime}\right)=u_{a} \cap \cap Q^{\prime}=a$. Whence $\cap\left(Q^{\prime}-q^{\prime}\right)=a$ contrary to the irredundancy of $Q^{\prime}$. Thus there is exactly one $q^{\prime} \in Q^{\prime}$ such that $q^{\prime} \geqq s$ and hence $\cap\left(Q^{\prime}-q^{\prime}\right) \geqq b$. According to the hypothesis of the lemma $q^{\prime}$ can be replaced by $q_{p}$ for some $p \in P$. Thus $a=q_{p} \cap \cap\left(Q^{\prime}-q^{\prime}\right)$. Hence $a \geqq q_{p} \cap b=\mathrm{U}(P-p)$ $\cap \cup(S)=\bigcup(S \wedge(P-p))$ and thus $S \wedge(P-p)=\varnothing$. Since $S \subseteq P$ it follows that $S=\{p\}$ and thus $b=\bigcup(S)=p>a=s \cap b$ contrary to hypothesis. Hence we may suppose that $s \cap b \neq a$. Next let $t^{*}=\bigcup(P-T)$ and suppose that $s \cap t^{*}=a$. Again there exists an irredundant decomposition $a=\cap Q^{\prime}$ such that $q^{\prime} \geqq s$ or $q^{\prime} \geqq t^{*}$ for each $q^{\prime} \in Q^{\prime}$. Also there is a unique $q^{\prime}$ such that $q^{\prime} \geqq s$. For this $q^{\prime}$ we have $\cap\left(Q^{\prime}-q^{\prime}\right) \geqq t^{*}$. Since by hypothesis $q^{\prime}$ can be replaced by $q_{p}$ for some $p \in P$ we get $a=\mathrm{U}(P-p) \cap U(P-T)=\mathrm{U}((P-p) \wedge(P-T))$. Thus $(P-p)$ $\wedge(P-T)=\varnothing$ and hence $T=P-p$. But then $u_{a}>\cup(P-p)=\bigcup T$ and hence $s=s \cap b$ contrary to $s \geqq b$. We may thus assume that $s \cap b \neq a$ and $s \cap t^{*} \neq a$. Furthermore $b \cap t^{*} \neq a$. For since $\cup S=b>s \cap b \geqq \cup(T)$ it follows that $\mathrm{U}(S-T)$ $>a$ and hence $b \cap t^{*} \geqq \bigcup(S-T)>a$. On the other hand $a=s \cap b \cap t^{*}$. Since if $s \cap b \cap t^{*} \geqq p>a$, we have $s \cap b \geqq p$ and hence $U T \geqq p$ which implies $a=U T$ $\cap U(P-T) \geqq p$ contrary to $p>a$. Thus the representation $a=s \cap b \cap t^{*}$ is irredundant and by Lemma 7.2, there exists an irredundant decomposition into irreducibles $a=\cap Q^{\prime}$ such that either $q^{\prime} \geqq s, q^{\prime} \geqq b$, or $q^{\prime} \geqq t^{*}$ for each $q^{\prime} \in Q^{\prime}$. By the argument given above there is a unique $q^{\prime}$ in $Q^{\prime}$ such that $q^{\prime} \geqq s$. By the replacement property we have $a=q_{p} \cap \cap\left(Q^{\prime}-q\right)$ for some $p \in P$. But then

$$
\begin{aligned}
a & \geqq U(P-p) \cap\left(b \cap t^{*}\right)=U(P-p) \cap U(S \wedge(P-T)) \\
& =U((P-p) \wedge[S \wedge(P-T)])=U(S-(T \vee p)) .
\end{aligned}
$$

Thus $S-T \vee p=\varnothing$ and thus $S=T \vee p$. Hence $b=\mathrm{U}(S)=\cup T \cup_{p}>\cup T$. But 
$b>s \cap b \geqq U T$. Thus $b>s \cap b$ contrary to hypothesis. The proof of the above statement is thus complete.

Now let $x$ be an arbitrary element of $u_{a} / a$, and let $c$ be a compact element such that $x \geqq c$. Then $U P_{a} \geqq x \geqq c$ and hence there exists a finite set $\left\{p_{1}, \cdots, p_{n}\right\}$ of elements of $P_{a}$ such that $p_{1} \cup \cdots \cup p_{n} \geqq c$. We show next that $p_{1} \cup \ldots \cup p_{n} / a$ satisfies the conditions of Lemma 7.3. It is clearly a finite dimensional semimodular lattice in which the unit element is a join of points. Now let $p_{1} \cup \ldots \cup p_{n}>t, p_{1} \cup \ldots \cup p_{n} \geqq w$ and $t \geqq w$ where $w$ is a join of elements of $P_{a}$. By Lemma 3.7, there exists $v$ such that $u_{a}>v, v \geqq t$ and $v \geq w$. By the first part of the proof we have $w>v \cap w=v \cap\left(p_{1} \cup \ldots \cup p_{n}\right) \cap w$ $=t \cap w$. Thus all of the conditions of Lemma 3.7 are satisfied and hence $p_{1} \cup \ldots \cup p_{n} / a$ is modular. Since $a \leq a \cup c \leq p_{1} \cup \ldots \cup p_{n}$ we conclude that $a \cup c$ is a join of points of $p_{1} \cup \ldots \cup p_{n} / a$ and hence is a join of the elements of $P_{a}$. But since $L$ is compactly generated, $x=\bigcup\{c \mid c \leqq x\}=\bigcup\{a \cup c \mid c \leqq x\}$ and thus $x$ is a join of elements of $P_{a}$. It follows that $u_{a} / a$ is a point lattice. Now if $x \cup y>y$ in $u_{a} / a$, by Lemma 3.7 there exists $s$ such that $u_{a}>s, s \geqq y$, and $s \geqq x \cup y$. Then $s \geq x$ and since $x$ is a join of points, the first part of the proof implies that $x>x \cap s$. But then $x>x \cap s=x \cap(x \cup y) \cap s=x \cap y$. Thus $u_{a} / a$ is lower semimodular and we conclude from Lemma 3.4 that $u_{a} / a$ is modular. Hence the proof of the lemma is complete.

Lemmas 7.1-7.4 give the following theorem.

THEOREM 7.1. A semimodular, compactly generated, atomic lattice has replaceable decompositions if and only if it is locally modular.

\section{REFERENCES}

1. G. Birkhoff, Lattice theory, rev. ed., Amer. Math. Soc. Colloquium Publications, vol. 25, New York, 1948.

2. R. P. Dilworth, Lattices with unique irreducible decompositions, Ann. of Math. (2) vol. 41 (1940) pp. 771-777. 299.

3. - The arithmetical theory of Birkhoff lattices, Duke Math. J. vol. 8 (1941) pp. 286-

4. - Ideals in Birkhoff lattices, Trans. Amer. Math. Soc. vol. 49 (1941) pp. 325-353.

5. - Note on the Kurosh-Ore theorem, Bull. Amer. Math. Soc. vol. 52 (1946) pp. 659663.

6. M. Hall, Distinct representatives of subsets, Bull. Amer. Math. Soc. vol. 54 (1948) pp. 922-926.

7. J. Hashimoto, Direct, subdirect decompositions and congruence relations, Osaka Math. J. vol. 9 (1957) pp. 87-112.

California Institute of Technology, Pasadena, California 